\title{
Subfascial Endoscopic Perforator Surgery: Retrospective Analysis of the First 50 Patients
}

\section{Carlos E Costa Almeida*}

Centro Hospitalar e Universitário de Coimbra - Hospital Geral (Covões), Portugal

*Corresponding author: CE Costa Almeida, Centro Hospitalar e Universitário de Coimbra - Hospital Geral (Covões), Rua José Carlos Gomes Pita, ${ }^{\circ} 2$, $2^{\circ}$ dto, 3040-193 Coimbra, Portugal, Tel: 00351 914722279; E-mail: carloscostaalmeida@yahoo.com

Rec. Date: Jul 28, 2015; Acc. Date: Sep 15, 2015; Pub. Date: Sep 21, 2015

Copyright: (C) 2015 Almeida CEC. This is an open-access article distributed under the terms of the Creative Commons Attribution License, which permits unrestricted use, distribution, and reproduction in any medium, provided the original author and source are credited.

\begin{abstract}
Introduction: Subfascial Endoscopic Perforator Surgery (SEPS) is a minimally invasive technique to treat incompetent perforating veins. Incompetent perforators are implicated in venous ulcer and in varicose veins recurrence. Complete closure of all perforating veins is the only predictor of ulcer healing. Analysis of our results is the objective of this study.
\end{abstract}

Methods: Fifty (50) patients with incompetent perforating veins were submitted to unilateral SEPS. Class CEAP C2-C6 patients were included. Ulcer-healing rate, ulcer recurrence rate, and complications rate were analysed.

Results: CEAP distribution was: C1-0; C2-5; C3-20; C4-11; C5-2; C6-12. An ulcer-healing rate of 92\% (11/12) was found at 6 months, with an average of 2.5 months until complete cicatrisation. Only one patient suffered an ulcer recurrence (9\%). Complications occurred in 4 patients $(8 \%)$, including 2 cases of surgical site infection (4\%).

Discussion: SEPS has a better ulcer-healing rate than other perforator ablation techniques. Our data match other studies data. The inclusion of patients submitted to SEPS plus GSV stripping and patients submitted only to SEPS can bring some doubts about the role of SEPS in ulcer healing. However from the patients treated only with SEPS, most of them had already been submitted to GSV removal in the past, and were now treated because of venous ulcer development and varicose veins recurrence.

Conclusion: These data support the importance of SEPS in perforating veins treatment and the hemodynamic role of perforators in venous ulcer development and varicose veins recurrence.

Keywords: Perforating veins; SEPS; Varicose veins recurrence; Venous hypertension; Venous ulcer

\section{Introduction}

Subfascial Endoscopic Perforator Surgery (SEPS) is a minimally invasive technique to treat incompetent perforating veins of lower limbs. Because incompetent perforators raise venous hypertension, they are implicated in venous ulcer and in varicose veins recurrence [1-3].

Although complete closure of all perforating veins is the only predictor of ulcer healing [1], some controversy exists about in which cases that treatment should be offered to patients. According to both the Society for Vascular Surgery and the American Venous Forum guidelines, there is indication to treat incompetent perforating veins in CEAP class C3-C6 (GRADE 2B), and they also recommend against the treatment of incompetent perforating veins in CEAP class C2 (GRADE 1B) [4]. However some authors recommend treating incompetent perforators in CEAP class $\mathrm{C} 2$ in the presence of varicose veins recurrence $[5,6]$.

Perforating veins incompetence is defined by retrograde (outward) flow lasting longer than 0.5 seconds or perforator with a diameter $\geq 3.5$ $\mathrm{mm}$ [4]. Between 1992 and 2008 SEPS became the technique of choice for perforators' ablation [4]. Since the emergence of ultrasound-guided percutaneous ablation techniques (thermal and chemical), which are attractive to doctors and patients, transformation is occurring in the treatment of perforators. However analysis of results from Percutaneous Ablation of Perforators (PAP), are showing that these new techniques may not have the same efficacy as SEPS according to ulcer healing and ulcer recurrence rates. Additionally duplex ultrasound will miss a mean of 2-3 incompetent perforators in one leg [2], meaning that these perforators will be left untreated when using percutaneous ablation techniques. With SEPS all perforators are found with the endoscope and all of them will be treated, being an advantage of this minimally invasive surgical technique.

Although there are no RCT in the literature comparing techniques, there are several reports talking about results for each one. Rhodes and Gloviczki report a mean ulcer-healing rate of $90 \%$ and a mean recurrence rate of $11 \%$ for SEPS [6]. Papers from Kiguchi [1] and Masuda [7] report an ulcer-healing rate of $59 \%$ to $67.6 \%$ and a recurrence of $32.4 \%$ for ultrasound-guided sclerotherapy. For Radiofrequency (RF) and Endovascular Laser Ablation (EVLA) techniques there are no studies in the literature reporting ulcer healing and ulcer recurrence rates.

The objective of this paper is to share with medical community our results and experience using SEPS to treat incompetent perforating veins of lower limbs, focusing ulcer-healing and recurrence rates. 
Page 2 of 3

\section{Methods}

A group of 50 patients submitted to unilateral SEPS were retrospectively analysed. Data on age, sex, CEAP class disease, previous Great Saphenous Vein (GSV) excision, and concomitant GSV removal were collected. All GSV were treated with surgery (high ligation plus stripping). None was treated with thermal ablation. A one-year follow-up period was accomplished with complication rates recorded. Ulcer-healing rate at 6 months and recurrence rate were analysed.

All patients had a duplex ultrasound done before surgery to diagnose the presence of incompetent perforating veins. The same surgical team operated all patients. The one-port technique was preferred, and for dissection, ligation and section of perforators a $5 \mathrm{~mm}$ LigaSure ${ }^{-}$was used. Venous Thromboembolism (VTE) prophylaxis was initiated $2 \mathrm{~h}$ before skin incision with Low Molecular Weight Heparin (LMWH). No antibiotic prophylaxis was used. Elastic bandages were applied at surgery, changing to elastic stockings class 2 at discharge date. Elastic therapy was continued for one week or until ulcer healing. No LMWH was used after discharge since all patients could walk properly and had no major risks for VTE. All patients were submitted to unilateral surgery.

\section{Results}

The mean age was 56.8 years old (minimum 37 years, maximum 84 years). From the 50 patients studied 32 were female (64\%) and 18 were male (36\%). CEAP class C2 to C6 patients were treated with SEPS if incompetent perforating veins were found at duplex ultrasound of lower limbs. CEAP distribution was: C1-0; C2-5; C3-20; C4-11; C52; C6-12.

Most patients (33) were submitted to SEPS plus GSV excision. Seventeen (17) patients were submitted only to SEPS, because fifteen (15) had had their GSV removed years before and two (2) had a normal GSV.

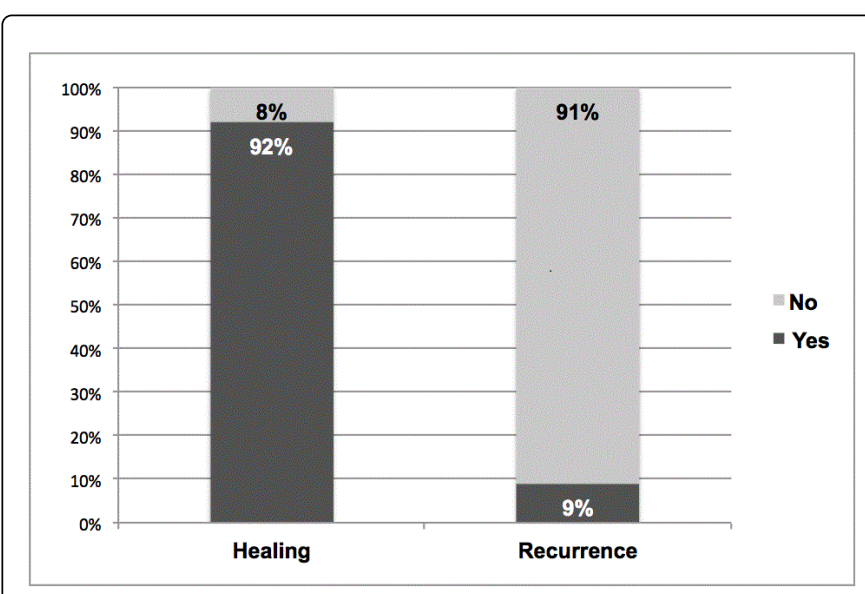

Graphic 1: Ulcer-healing rate (92\%) and ulcer recurrence rate (9\%) at 6 months of follow-up.

An ulcer-healing rate of $92 \%(11 / 12)$ was found at 6 months, with an average of 2.5 months until complete cicatrisation. Only one patient suffered an ulcer recurrence (9\%), occurring 11 weeks after healing (Graphic 1). From the 15 patients group who had their GSV removed years before, 7 were CEAP class C6 disease, and after SEPS all ulcers healed. Complications occurred in 4 patients (8\%), including 2 cases of surgical site infection (4\%), one leg thrombophlebitis (2\%), and a case of leg paraesthesia (2\%).

\section{Discussion}

For performing SEPS we prefer the one-port technique, using a device specially designed for this procedure, eliminating the "swordfighting" issue of the instruments. However this endoscope is no longer available in the market, so we are now using the two-port technique, described by O'Donnell in the US. To make SEPS easier we are only using a $5 \mathrm{~mm}$ LigaSure $^{\oplus}$ to dissection, ligation and section, eliminating the need to change instruments during surgery. The only major difficulty of the two-port technique is $\mathrm{CO}_{2}$ insufflation and subfascial space creation, which is easier with the one-port technique giving the surgeon a better working space. For the one-port technique we use a $19 \mathrm{mmHg}$ pressure and for the two-port a $25 \mathrm{mmHg}$ pressure, and even so the working space is better when using the one-port endoscope.

According to the clinical practice guidelines of the Society for Vascular Surgery and the American Venous Forum there is indication to treat incompetent perforators in CEAP class C3-C6 disease [4]. However, because incompetent perforating veins of the lower limbs are responsible for varicose ulcer development and varicose veins recurrence, the author included $\mathrm{C} 2$ patients with incompetent perforators in the group with indication to SEPS.

Although only 12 patients with C6 disease were studied, the author reports an ulcer-healing rate of $92 \%$ at six months and a recurrence rate of 9\%, matching other authors' data. In his book "Phlebology", Ramelet reports an $88 \%$ ulcer-healing rate, and recurrence rate of $13 \%$ and $18 \%$ for C5 and C6 disease respectively [8]. Rhodes and Gloviczki report a mean ulcer-healing rate of $90 \%$ and a mean recurrence rate of $11 \%$ for SEPS [6]. One drawback of this analysis, like all other similar trials, is the inclusion of patients submitted to SEPS plus GSV stripping and patients submitted only to SEPS, which can bring some doubts about the role of SEPS in ulcer-healing. However from the 17 patients group treated only with SEPS, 15 had already been submitted to GSV removal (high ligation plus stripping) in the past, and were now treated because of venous ulcer development (7/15) and varicose veins recurrence (8/15). This fact supports the hemodynamic importance of perforating veins in venous ulcer and in varicose veins recurrence, validating the indication for perforating veins' treatment.

In this study surgery (high ligation plus stripping) was the treatment of choice to treat GSV, and none was treated with thermal ablation techniques, including Endovascular Laser Ablation (EVLA) and Radiofrequency Ablation (RFA). Although thermal ablation techniques have some advantages (less post-operative pain, less bruising, faster recovery and return to work), medium-term follow-up trials show similar clinical and hemodynamic outcomes comparing to surgery [4]. Additionally long-term results are lacking on thermal ablation techniques. According to data now available, using EVLA or RFA to treat GSV wouldn't be expected to change results of this study nor the importance of incompetent perforating veins treatment.

Complications occurred in $8 \%$ of patients, with a SSI rate of only $4 \%$. This is a great advantage of SEPS comparing to open surgery, which has a reported wound complication rate near $25 \%$. Gloviczki also reports a complication rate of 5\% with SEPS [6]. A case of leg paraesthesia is reported (2\%), however this complication was probably due to saphenous nerve injury during GSV stripping and not because 
Citation: Almeida CEC (2015) Subfascial Endoscopic Perforator Surgery: Retrospective Analysis of the First 50 Patients. J Vasc Med Surg 3: 221. doi: $10.4172 / 2329-6925.1000221$

Page 3 of 3

of SEPS. This complication was temporary without any specific treatment.

\section{Conclusion}

Incompetent perforating veins are responsible for venous ulcer and varicose veins recurrence. Because of that C2-C6 patients with incompetent perforators were included in this analysis. SEPS has an ulcer-healing rate of $92 \%$ and an ulcer recurrence rate of $9 \%$. These data support the importance of this technique in perforating veins treatment, and the hemodynamic role of perforators in venous ulcer development and varicose veins recurrence.

\section{References}

1. Kiguchi MM, Hager ES, Winger DG, Hirsch SA, Chaer RA, et al. (2014) Factors that influence perforators thrombosis and predict healing: perforator sclerotherapy for venous ulceration without axial reflux. J Vasc Surg 59: 1368-1376.

2. Steve Elias (2009) Percutaneous ablation of perforating veins. In: Gloviczki P. Handbook of venous disorders. (3rd edtn). Hodder Arnold, London. Pp: 536-544.
3. Costa Almeida CE (2014) Treatment of perforating veins - Review of techniques. Revista Portuguesa Cirurgia 31: 27-33.

4. Gloviczki P, Comerota AJ, Dalsing MC, Eklof BG, Gillespie DL, et al. (2011) The care of patients with varicose veins and associated chronic venous disease: clinical practice guidelines of the Society for Vascular Surgery and the American Venous Forum. J Vasc Surg 53: 2S-48S.

5. O'Donnell TF (2010) The role of perforators in chronic venous insufficiency. Phlebology 25: 3-10.

6. Rhodes JM, Kalra M, Gloviczki P (2009) The management of incompetent perforating veins with open and endoscopic surgery. In: Gloviczki P. Hanbook of venous disorders. (3rd edtn). Hodder Arnold, London. Pp: 523-535.

7. Masuda EM, Kessler DM, Lurie F, Puggioni A, Kistner RL, et al. (2006) The effect of ultrasound-guided sclerotherapy of incompetent perforator veins on venous clinical severity and disability scores. J Vasc Surg 43: 551-557.

8. Ramelet AA (2008) Treatment of venous ulcers. Phlebology. 5: 435-479. 